\title{
THE EFFECTS OF A VOCAL LOADING TASK ON THE ACOUSTIC AND ELECTROMYOGRAPHIC MEASUREMENTS OF VOCAL FATIGUE
}




\section{ACKNOWLEDGMENTS}

I would like to thank my honors thesis panel for their support and insightful feedback throughout this process. Thank you to Dr. Watts for being my project supervisor, and thank you to Mrs. Lambert and Dr. Dennis for participating on the review panel; it has been a privilege to work with all of you this past year. Thanks to you, this research was made possible in the hopes of improving quality of care for future voice clients; I truly could not have accomplished this without all your help. 


\section{INTRODUCTION}

Voice production is an essential part of communicating effectively. When the voice is overused, however, the results can be severe. Vocal fatigue can be a negative consequence of voice overuse (Welham \& Maclagan, 2003). Clients with vocal fatigue experience symptoms such as pain, difficulty speaking, and shortness of breath (Kostyk \& Rochet, 1998). Many studies have investigated vocal fatigue using acoustic assessments, but few have compared those findings to physiological correlates such as electromyographic measurements (Lin, 2017). A direct way of utilizing electromyography is the hooked-wire method, which is highly invasive and painful for the client (Ferster \& Hu, 2017). Surface electromyography, or SEMG, could allow the muscles involved in voice production to be measured directly in a noninvasive manner; few reports exist that investigate using this as a tool for measuring vocal fatigue (Khoddami et al, 2017). In all, the possibility of using SEMG in addition to acoustic measurements could help speech-language pathologists assess vocal fatigue more effectively.

\section{LITERATURE REVIEW}

Phonation is defined as "the quasiperiodic vibration of the vocal folds for production of speech voicing" (Seikel, Drumright, \& Seikel, 2013). The vocal folds are small, paired structures made of elastic mucosal membranes, muscles, and tendons; they are about 17-24 millimeters long in men and 13-17 millimeters long in women (Borden, Harris, \& Rafael 2011). The vocal folds are located just above the trachea inside of the larynx, a complex structure of cartilage, muscle, and bone (Seikel et al, 2013). Muscle activity is responsible for bringing the vocal folds together so that voice production, or phonation, is accomplished.

There are several theories for how phonation occurs. One of the most widelyaccepted is the aerodynamic myoelastic theory in which air pressure below adducted vocal folds forces them apart, and then they come back together because of elasticity and aerodynamic forces (Borden et al, 2011). Among the most important elements of phonation is muscle activity, which allows the vocal folds to approximate at midline so that aerodynamic forces can exert influence on them.

The larynx consists of intrinsic muscles, which have both origin and insertion inside the larynx, and extrinsic muscles, which have one attachment in the larynx and one attachment outside of the larynx; the intrinsic muscles 
manipulate the vocal folds, and the extrinsic muscles move the larynx (Seikel et al, 2013). The muscles utilized during voice production are prone to fatigue. However, it has been stated that "the field of voice research currently lacks a universally accepted definition of vocal fatigue" (Welham \& Maclagan, 2003). One definition is the undesirable symptoms that occur when the vocal folds are damaged by frequent use, "indicating undesirable or unexpected changes in the functional status of the laryngeal mechanism" (Welham \& Maclagan, 2003). This is a problem especially for those in occupations that require prolonged use of the voice, such as teaching, singing, and acting (Welham \& Maclagan, 2003). The most frequent client-reported symptoms of vocal fatigue include hoarseness, breathiness, less intensity, running out of breath during speech, neck tension, painful swallows, and discomfort (Kostyk \& Rochet, 1998).

In general, clients with vocal fatigue must exert more effort to speak. At the laryngeal level, this manifests in changes in timbre (voice quality), intensity, fundamental frequency, inflammation and dryness in the larynx (Guzmán et al, 2013). More serious issues such as lesions in the vocal folds can also occur with overuse of the voice (Guzmán et al, 2013). Many vocal fatigue clients could also suffer from submucosal hemorrhages in the vocal folds or other acute laryngeal issues (Hunter \& Banks, 2017). Other than simply overuse or misuse of the voice, the underlying cause of vocal fatigue can be related to neurological impairments or maladaptive vocal technique (Stemple, Stanley, \& Lee, 1995).

A measurement tool which has been used to examine vocal muscular activity is electromyography. One example is hooked-wire laryngeal electromyography (LEMG) in which needles are inserted into major laryngeal muscles to measure their activity (Ferster \& Hu, 2017). This method has shown to be successful in assessing voice disorders such as vocal fold paralysis (Maamary et al, 2017). The client undergoing this invasive procedure, however, must be awake to phonate for measurements and may experience pain from the needle insertions (Ferster \& Hu, 2017). A less invasive procedure is surface electromyography (sEMG) in which peak muscle activity and contraction duration is measured by surface electrodes placed on the skin (Khoddami et al, 2017). In one cross-sectional study, it was reported that sEMG was a "highly reliable tool" to measure muscle laryngeal activity during phonation in clients with dysphonia when electrodes were placed on the skin above the larynx (Khoddami et al, 2017).

Another way to examine vocal activity is acoustic measurement. Acoustic measurement is the most widely-used and least-invasive method of assessing the voice (Lin, 2017). Acoustic measurements are obtained when a voice sample is recorded and analyzed with a computer program to identify specific 
frequency and amplitude characteristics within the acoustic signal (Carding, 2009). This is an objective measure of voice that does not rely on subjective perceptual impressions (Hanson, 1997). Variables typically assessed with acoustics include the harmonics- to-noise ratio, fundamental frequency (fo), jitter, and shimmer (Lopes et al, 2017). Acoustic measures of vocal activity, especially of vocal fatigue, have been controversial among researchers; accurate biofeed back measurements would require very invasive procedures(Guzmán et al, 2013). However, there is no current consensus on which acoustic measures best relate to vocal fatigue.

Vocal loading is described as extensive voice use that includes a warmup period, the task, the following vocal fatigue, and recovery (Kelchner et al, 2006). Vocal loading tasks are more commonly used to compare voice data for before-and-after purposes (Kelchner, 2006 measurements alone do not present sufficient data (Guzmán et al, 2013). High-intensity vocal use over a long time creates vocal fatigue, so the vocal loading task mimics this action (Whitling, 2017a). Tasks can vary with how loud the client must speak, how long this occurs, and what is read aloud (Kelchner, 2006). A reading task is the most common way to achieve this (Kelchner, 2006). The client's self-ratings of his or her voice quality and comfort are crucial to comparing results before and after the task (Kelchner, 2006). In many vocal loading tasks, the clients must make themselves heard over recorded ambient noise; the intensity of the noise can reach $85 \mathrm{~dB}$ SPL (Whitling, 2015). The task can be measured using digital analysis programs wherein the live voice is analyzed with computer software (Vertigan, 2017). Usually, the clients who will perform the task are asked to refrain from voice overuse and caffeine for 24 hours before the task (Kelchner, 2006). Vocal loading tasks have not been shown to create permanent voice damage, but acute anterior chinks in the vocal chords have been reported (Whitling, 2015).

Surveys are often used to measure the condition of a client's voice; this method has high validity because the client presents and reports on his or her own voice. Several standardized surveys are also available, including the Buffalo Voice Scale and the Consensus of Auditory Perceptual Evaluation of Voice scale; these reports measure strain and breathiness among other symptoms (Carding, 2009). In studies that tested the reliability of the Vocal Performance Questionnaire, the Voice Handicap Index, and the Voice Symptom Scale, all three surveys demonstrated "excellent internal consistency and repeatability." Client-reported surveys can include marking a point on a spectrum between "not at all" and "extremely" regarding levels of fatigue, comfort, and control of the voice; these responses are then converted into percentages (Bottalico, 2015). 
Other surveys include filling in statements on a scale of $O$ to 5 (none to very high); these report symptoms of hoarseness, stress, and fatigue (Whitling, 2017b). Some surveys require a trained listener, that is, a speech-language pathologist with at least one year of experience in the area of voice disorders, to measure the client's voice (Hanson, 1997).

\section{RESEARCH QUESTIONS}

In summary, laryngeal muscles are responsible for bringing the vocal folds together for voice production. When these muscles are overused, the speaker experiences symptoms of vocal fatigue like breathiness, hoarseness, pain upon speaking, and tension; these may have an underlying cause that can lead to more severe symptoms. A speech pathologist can directly measure the muscles of a fatigued voice through electromyography. The hooked-wire method is valid, yet it may cause the client severe discomfort; the surface method is less invasive, but it is not fully researched as a way to measure laryngeal muscles. Acoustic measurements of the voice allow speech pathologists to examine the frequency and amplitude components of a signal, but this type of measurement alone is not sufficient for vocally-fatigued clients.

The purpose of this study was to determine the effect of a vocal loading task on submandibular muscular activity and acoustic measures of vocal function. Surface electromyography (sEMG) directly assessed muscle activation, and these findings were compared with indirect acoustic measures from voice recordings. If these measurements accurately measured muscle fatigue after a vocal loading task, the results may help speech language pathologists better assess vocal fatigue in their clients. The following research questions were investigated:

1. As measured with surface electromyography, would muscle activity increase significantly post-vocal loading in comparison to pre-vocal loading?

2. Would cepstral peak prominence indicate a significant decrease in vocal fold vibration periodicity post-vocal loading?

3. Would low to high frequency spectral ratio results indicate a significant increase of air escaping between adducted vocal folds post-vocal loading? 


\section{METHODOLOGY}

\section{Participants}

There were 14 participants in this study; these were young, healthy adults recruited from the undergraduate and graduate student population at Texas Christian University in Fort Worth, Texas. The inclusionary criteria were that the participants must be 18 to 25 years of age. The exclusionary criteria were that the participants must not have a current voice disorder or history of voice disorders, must pass a hearing screening in the Miller Speech and Hearing Clinic on the day of data collection, must have no reports of throat irritation, must not have had professional voice or singing training within the past 3 years, and must not have any past or present neurological disease.

\section{Instrumentation/Tools}

The participants completed the vocal loading task by reading aloud at $75 \mathrm{~dB}$ or louder from the book Harry Potter and the Sorcerer's Stone by J.K. Rowling. This book was selected for the following reasons: thirty minutes of vocal loading could be completed without finishing the entire book, the reading level is easy enough that reading difficulty would not be a limitation for college-age participants, and the popularity of book among the participant demographic would motivate the reader to participate. To ensure that participants were producing the vocal task at an intensity level greater than typical conversation, a visual feedback tool called HiVOLT (www.voiceaerobics. com) was used. This tool produces a flashing light when sound amplitude is $75 \mathrm{~dB}$ or greater, and the flash continues until sound amplitude decreases below that level. To acquire measures of laryngeal muscular activity, the extrinsic laryngeal elevators were assessed using the Swallowing Systems Lab surface electromyography (sEMG) system (Pentax Medical, Montvale, NJ). This system consists of a recording electrode placed on the surface of the skin directly above the submandibular laryngeal elevator muscles (geniohyoid, mylohyoid, anterior digastric). Contraction amplitude and contraction duration can be calculated from the recorded sEMG waveforms, which are acquired using a computer connected to the surface electrode. Acoustic recordings and measurements were obtained using the Computerized Speech Lab and associated Analysis of Dysphonia in Speech and Voice software (Pentax Medical, Montavale, NJ). 
Many of the voice surveys researched were designed for clients with existing voice problems and their effects on daily activities. Surveys created for vocal loading tasks often covered the client's own perception of fatigue, stress, discomfort, and similar factors. Because of this, the researcher gave the participants a ten-question survey inspired by the Voice Symptom Scale and Vocal Handicap Index. The participant received the questions on a piece of paper, and each answer will be on a scale of 1-5 with 1 meaning "not at all" and 5 meaning "a lot or very much." The questions were as follows: (1) My throat or neck hurts; (2) I feel tense; (3) My throat feels dry or creaky; (4) I have to clear my throat; (5) It would hurt if I swallowed right now; (6) I feel hoarse; (7) I am feeling tired; (8) I feel out of breath; (9) I don't want to use my voice for the rest of the day; (10) I found the task difficult to complete.

\section{Procedures}

All participants gave consent to participate in the study by completing the provided consent form. Once consent was given, the participant had a hearing screening at the Miller Clinic in an audiology booth to ensure normal hearing. The portable audiometer used in this screening was the Amplivox 270. The participant passed the screening if the air conduction hearing threshold was at $25 \mathrm{~dB}$ or lower at frequencies of $500 \mathrm{~Hz}, 1000 \mathrm{~Hz}, 2000 \mathrm{~Hz}$, and $4000 \mathrm{~Hz}$. If a potential participant did not pass, he or she was dismissed from the rest of the process. After passing the hearing screening, the participant was given verbal instructions regarding how the sEMG electrodes would be placed and used, what would be recorded for acoustic analysis, and how to complete the vocal loading task. The participant was then allowed to ask any questions before beginning data collection. Next, the sEMG electrodes were placed on the participant. The skin surface was cleaned with an alcohol wipe (MEDI-PAK 70\% Isopropyl Alcohol) prior to application of the electrode to remove skin oils. The sEMC electrode is designed as a self-adhesive patch consisting of two recording and one ground electrode. This was placed centrally $1 \mathrm{~cm}$ below the inferior rim of the mental protuberance of the mandible; this placement was to insure that the electrodes were over the submandibular laryngeal elevator muscles. The device then collected the SEMG waveforms of muscle activity during vocal tasks, and the electrodes remained on the participant in order to save time on reapplication. First, the client was provided 1 tablespoon of vanilla or chocolate Snack Pack pudding and given instructions to swallow the pudding as hard as he or she could. 
Then, the client was prompted to sustain the vowel /a/ at a comfortable level for approximately 5 seconds; this was done three times. Next, the client read aloud at a normal speaking level, "We were away a year ago," three times. Finally, the client read a paragraph called "The Rainbow Passage" one time aloud at a normal speaking level from another piece of paper. The researcher then placed a head-mounted microphone, in which the receiver was approximately $3 \mathrm{~cm}$ from the left corner of the mouth, on the client's head. The microphone was connected to a computer with the Computerized Speech Lab application (CSL) with the Analysis of Dysphonia in Speech and Voice (ADSV) software. First, the client was prompted to sustain the vowel "a" at a comfortable level for approximately 5 seconds; this was done three times.

Next, the client read aloud at a normal speaking level, "We were away a year ago," three times. After this, the client read "The Rainbow Passage" one time aloud at a normal speaking level from another piece of paper. Finally, the client glided from the lowest pitch to the highest pitch on the vowel "e." All of these recordings were analyzed later for the acoustic measurements of cepstral peak prominence ( $\mathrm{CPP}$, in $\mathrm{dB}$ ), low-to-high frequency spectral ratio $(\mathrm{L} / \mathrm{H}$ ratio), and semitone range (for the gliding task only). The first measurement represents the periodicity of the vocal fold vibrations, the second represents the amount of noise, or air escaping between the vocal folds, and the third represents the range between the lowest and highest frequencies of a voice sample.

Once the recordings were collected, the participant received a clipboard with Harry Potter and the Sorcerer's Stone clipped open to the first page and the Hi-VOLT attached to the top of the clipboard. Once prompted to begin, the participant read the book aloud; the participant was required to read loud enough for the Hi-VOLT to flash at least once every 5 seconds, which was monitored by the researcher. If the patient failed to produce utterances at the $>75 \mathrm{~dB}$ level every five seconds, they were verbally prompted by the investigator to increase their loudness with the phrase "Louder, please." After thirty minutes elapsed, as measured on a mobile phone timer application, or the participant verbally reported symptoms of throat pain, the participant was prompted to stop reading aloud; the acoustic measurements were immediately collected via microphone just as before. After this, the sEMC electrodes, still on the participant, collected the waveform measurements once more. All vocal tasks were repeated during these procedures. Finally, after the electrodes and microphone were removed, the participant completed the provided self-report voice survey on paper before leaving the clinic. 


\section{Analysis}

The independent variable in this study is measurement period (pre-vocal loading and post-vocal loading). The dependent variables were: (a) total score on the fatigue questionnaire; (b) CPP of the vowel; (c) CPP of the sentence; (d) CPP of the paragraph; (e) L/H ratio of the vowel; (f) $\mathrm{L} / \mathrm{H}$ ratio of the sentence; $(\mathrm{g}) \mathrm{L} / \mathrm{H}$ ratio of the paragraph; (h) semitone range in the gliding task; (i) percentage of maximum muscle activity (in comparison to the swallowing task) of the vowel; (j) percentage of maximum muscle activity of the sentence; (k) percentage of maximum muscle activity of the paragraph; (I) average acoustic amplitude of the vowel; $(\mathrm{m})$ average acoustic amplitude of the sentence; and $(\mathrm{n})$ average acoustic amplitude of the paragraph. The research questions were addressed statistically using paired t-tests to compare pre-vocal loading to post-vocal loading measures. Because this is an exploratory project with a large number of statistical tests on means, the alpha level was set to 0.025 for all comparisons in order to control for Type 1 error.

\section{Results}

Table 1 displays descriptive statistics for each acoustic and electromyographic dependent variable for the pre-reading task and post-reading task. A consistent trend emerged in this data. At the vowel, sentence, and "The Rainbow Passage" level, participants increased their cepstral peak prominence and decreased their low to high spectral ratio after the reading task. The semitone range during a glide from lowest to highest pitch decreased after the reading task. In addition, at the vowel, sentence, and "The Rainbow Passage" level, the participants decreased the percentage of muscle activity in comparison to maximum level. Participants also increased their acoustic amplitude after the reading task at the vowel, sentence, and "The Rainbow Passage" level.

In order to control for Type 1 error due to a large number of statistical tests on means, the alpha level was set to 0.025 for all t-test comparisons. Table 2 shows the results of paired-samples t-tests applied to each pre-reading and post-reading mean. Measurements that were found to be significantly different when comparing pre-reading task to postreading task were the changes in cepstral peak prominence at the sentence level, the changes in cepstral peak prominence at "The Rainbow Passage" level, and the changes in acoustic amplitude at "The Rainbow Passage" level. The changes in all other measurements did not reach statistical significance; however, their trends were consistent. To test for the effect of sex on acoustic measurements, a MANOVA was applied to the data with sex as the independent variable, but this yielded a probability value of 0.258 , so sex did not significantly affect the results. 
TABLE 7. Means and standard deviations of dependent variables.

\begin{tabular}{|l|l|c|c|c|}
\hline & $\begin{array}{l}\text { Task- } \\
\text { Measurement- } \\
\text { Pre or Post }\end{array}$ & Mean & N & $\begin{array}{c}\text { Std. } \\
\text { Deviation }\end{array}$ \\
\hline Pair 1 & V-CPP-Pre & 11.93 & 14 & 2.24 \\
\hline & V-CPP-Post & 12.37 & 14 & 2.08 \\
\hline Pair 2 & V-LH-Pre & 43.10 & 14 & 3.76 \\
\hline & V-LH-Post & 42.09 & 14 & 3.10 \\
\hline Pair 3 & S-CPP-Pre & 5.82 & 14 & 1.27 \\
\hline & S-CPP-Post & 6.72 & 14 & 0.76 \\
\hline Pair 4 & S-LH-Pre & 34.49 & 14 & 4.08 \\
\hline & S-LH-Post & 33.38 & 14 & 3.15 \\
\hline Pair 5 & R-CPP-Pre & 5.93 & 14 & 0.71 \\
\hline & R-CPP-Post & 6.47 & 14 & 0.70 \\
\hline Pair 6 & R-LH-Pre & 32.74 & 14 & 3.08 \\
\hline & R-LH-Post & 32.09 & 14 & 3.39 \\
\hline Pair 7 & G-Semi-Pre & 17.55 & 14 & 6.46 \\
\hline & G-Semi-Post & 17.43 & 14 & 5.94 \\
\hline Pair 8 & V-sEMG-Pre & 0.23 & 13 & 0.25 \\
\hline & V-sEMG-Post & 0.07 & 13 & 0.08 \\
\hline Pair 9 & S-sEMG-Pre & 0.23 & 13 & 0.20 \\
\hline & S-sEMG-Post & 0.12 & 13 & 0.12 \\
\hline Pair 10 & R-sEMG-Pre & 0.24 & 13 & 0.24 \\
\hline & R-sEMG-Post & 0.17 & 13 & 0.17 \\
\hline Pair 11 & V-Amp-Pre & 74.00 & 14 & 4.04 \\
\hline & V-Amp-Post & 75.45 & 14 & 3.41 \\
\hline Pair 12 & S-Amp-Pre & 74.60 & 14 & 3.11 \\
\hline & S-Amp-Post & 75.78 & 14 & 2.65 \\
\hline Pair 13 & R-Amp-Pre & 71.45 & 14 & 1.96 \\
\hline & R-Amp-post & 73.64 & 14 & 2.27 \\
\hline & \multicolumn{3}{|l}{} \\
\hline
\end{tabular}

Note: $\mathrm{V}=$ Vowel, $\mathrm{S}$ = Sentence, $\mathrm{R}=$ "The Rainbow Passage," $\mathrm{G}=$ Glide, CPP = Cepstral Peak Prominence, $\mathrm{LH}=$ Low to High Spectral Ratio, sEMG = sEMG percentage of maximum muscle effort used in task, Amp = Average Acoustic Amplitude. Because one male participant had facial hair that hindered sEMG measurements, he was not included in sEMG data, but was included in acoustic data. 


\section{TABLE 2. Paired Samples T-Test}

\begin{tabular}{|l|l|l|l|l|l|}
\hline & & $\begin{array}{l}\text { Paired } \\
95 \% \\
\text { Confidence } \\
\text { Interval of } \\
\text { the Upper } \\
\text { Limit }\end{array}$ & & & \\
& & t & df & Sig. (2-Tailed) \\
\hline Pair 1 & V-CPP-Pre \& -Post & 0.52 & -0.99 & 13 & 0.343 \\
\hline Pair 2 & V-LH-Pre \& -Post & 2.90 & 1.16 & 13 & 0.268 \\
\hline Pair 3 & S-CPP-Pre \& -Post & -0.42 & -4.03 & 13 & $\mathbf{0 . 0 0 1}$ \\
\hline Pair 4 & S-LH-Pre \& -Post & 2.62 & 1.61 & 13 & 0.132 \\
\hline Pair 5 & R-CPP-Pre \& -Post & -0.11 & -2.73 & 13 & $\mathbf{0 . 0 1 7}$ \\
\hline Pair 6 & R-LH-Pre \& -Post & 1.13 & 1.13 & 13 & 0.278 \\
\hline Pair 7 & G-Semi-Pre \& -Post & 0.14 & 0.14 & 13 & 0.892 \\
\hline Pair 8 & V-sEMG-Pre \& -Post & 2.31 & 2.31 & 12 & 0.040 \\
\hline Pair 9 & S-sEMG-Pre \& -Post & 1.86 & 1.86 & 12 & 0.087 \\
\hline Pair 10 & R-sEMG-Pre \& -Post & 1.72 & 1.72 & 12 & 0.112 \\
\hline Pair 11 & V-Amp-Pre \& -Post & -1.28 & -1.28 & 13 & 0.225 \\
\hline Pair 12 & S-Amp-Pre \& -Post & -1.56 & -1.56 & 13 & 0.143 \\
\hline Pair 13 & R-Amp-Pre \& -Post & -4.73 & -4.73 & 13 & $<\mathbf{0 . 0 0 1}$ \\
\hline
\end{tabular}

\section{DISCUSSION}

The purpose of this study was to determine the effect of a vocal loading task on submandibular muscular activity and acoustic measures of vocal function. The first research question asked was: As measured with surface electromyography, would muscle activity increase significantly post-vocal loading in comparison to pre-vocal loading? The data trend was consistent for sEMC measurements across all speech stimuli. After the vocal loading task, sEMG amplitude decreased for sustained vowel, sentence, and "The Rainbow Passage" productions.

However, the decrease did not reach statistical significance for any condition, and therefore it cannot be said that the vocal loading task had an effect on sEMC amplitude of the submandibular muscles.

The second research question asked was: Would cepstral peak prominence indicate a significant decrease in vocal fold vibration periodicity post-vocal loading? Results of the study indicated that vocal loading had a significant effect on measures of CPP in the sentence and "The Rainbow Passage" stimuli. Specifically, CPP increased after the vocal loading task in both conditions. Typically, larger measures of CPP reflect greater phonation periodicity, whereas our hypotheses were that the vocal loading task would elicit a decrease 
in periodicity. Vocal intensity can influence measures of CPP, and so we investigated this factor for all speech stimuli. Vocal intensity did significantly increase after the vocal loading task in "The Rainbow Passage," which may explain the increase in CPP. While the mean vocal intensity also increased in the sentence productions, this did not reach statistical significance. It may be that this study did not have adequate statistical power to detect that difference.

The third research question asked was: Would low to high frequency spectral ratio results indicate a significant increase of air escaping between adducted vocal folds post-vocal loading? The results of this study found that a vocal loading task had no effect on measures of $\mathrm{L} / \mathrm{H}$ spectral ratio. Typically, changes in this measure reflect changes to the degree of glottal closure during phonation. While the trend was for $\mathrm{L} / \mathrm{H}$ spectral ratio to decrease in each speaking condition, the difference in pre- to post measures was not great enough to reach statistical significance. This may suggest that the vocal loading task did not fatigue intrinsic laryngeal muscles enough to cause glottal incompetence. Alternatively, the study may not have had enough statistical power to detect an effect.

As shown in Table 1, there was a consistent change in acoustic and electromyographic measurements from pre-vocal loading to post-vocal loading. However, only measures of CPP in the sentence and "The Rainbow Passage" tasks reached statistical differences after the vocal loading task. This was likely explained by increases in vocal intensity, although the increase from pre- to post vocal intensity was only statistically significant for "The Rainbow Passage."

Although it is likely that this study did not have adequate statistical power, the data trends in the descriptive statistics are interesting and provide rationale for further research. After the vocal loading task, a combination of lowered muscle activity, higher periodicity of vocal fold vibration, more air escaping between adducted vocal folds, and higher acoustic intensity may indicate that increased vocal intensity could be a compensatory adaptation for an individual with a fatigued voice. In other words, an individual may exert more vocal effort after the vocal loading task due to muscle fatigue. Semitone range decreased slightly on average, which suggests that the full range of the voice was hindered due to the vocal loading task. In addition, on average, all participants in the study reported more severe levels of common vocal fatigue symptoms in comparison to before the vocal loading task. The only question whose responses remained static before and after the task was the phrase "I feel tired"; this could be due to the phrase's ambiguity. One participant may have felt sleepy from an early morning while another participant may have felt tiredness only in the voice. Nonetheless, the survey results indicate that the vocal loading 
task was successful in inducing vocal fatigue in a controlled setting.

\section{LIMITATIONS OF THE STUDY}

A number of factors should be considered for improving further research in this area of voice assessment. The small number of significant measurements after analysis suggest that the study was underpowered; a high number of participants could increase the significance of more measurements. The postvocal loading measurements may have been acquired too soon to reflect tissue changes. It is likely that edema and/or inflammation that occurs after phonotrauma takes time to develop. Future studies should also acquire measurements between thirty minutes to an hour after the vocal loading task to test for this theory. All participants caused the High-VOLT to flash in a manner of ways; some participants could read loudly enough for the device to flash without stopping, but some participants had to be reminded more frequently to read louder. More strict criteria could be put in place for this kind of reading task in order to standardize it for all participants. In addition, all participants spent at least an hour in the clinic; only one researcher would conduct data collection at a time for each participant; additional researchers in the room may cut down on the amount of time required of the participant.

\section{CONCLUSIONS}

Two different kinds of measurements-acoustic and electromyographicwere used to measure healthy, young adults' voices before and after a vocal loading task. Results indicated that increased vocal effort was reflected in greater acoustic vocal intensity translating to significantly increased measures of CPP in productions of "The Rainbow Passage." These results suggest that vocal effort may be reflected in a louder voice, which is perhaps a compensatory adaptation secondary to vocal fatigue. Self-assessment surveys further supported the presence of vocal fatigue after the reading task. In all, additional studies that investigate voice assessment in relation to vocal fatigue will increase the amount of significant evidence for better treatment of individuals with voice issues. 


\section{REFERENCES}

Borden, G. J., Harris, K. S., \& Raphael, L. J. (2007). Speech Science Primer (5th ed.). Philadelphia: Lippincott Williams \& Wilkins.

Bottalico, P., Graetzer, S., \& Hunter, E. J. (2015). Effects of voice style, noise level, and acoustic feedback on objective and subjective voice evaluations. The Journal of the Acoustical Society of America, 138(6). http://doi. org/10.1121/1.4936643

Carding, P. N., Wilson, J. A., MacKenzie, K., \& Deary, I. J. (2009). Measuring voice outcomes: State of the science review. The Journal of Laryngology and Otology, 123(8). http://doi.org/10.1017/S0022215109005398

Ferster, A. \& Hu, A. (2017). Perceptions of pain of laryngeal electromyography. The Laryngoscope. 128(4), 896-900. http://doi.org/10.1002/lary.26860

Giovanni, A., Revis, J., \& Triglia, J. (1999). Objective aerodynamic and acoustic measurement of voice improvement after phonosurgery. The Laryngoscope, 109(4), 656-660. http://doi.org/10.1097/00005537-19990400000026

Guzmán, M., Malebrán, M. C., Zavala, P., Saldívar, P., \& Muñoz, D. (2013). Acoustic changes of the voice as signs of vocal fatigue in radio broadcasters: Preliminary findings. Acta Otorrinolaringológica Española, 64(3), 176. http://doi.org/10.1016/j.otoeng.2013.06.011

Hanson, D. G., Jiang, J. J., Chen, J., \& Pauloski, B. R. (1997). Acoustic measurement of change in voice quality with treatment for chronic posterior laryngitis. The Annals of Otology, Rhinology \& Laryngology, 106(4). https://doi. org/10.1177/000348949710600403

Kelchner, L. N., Toner, M. M., \& Lee, L. (2006). Effects of prolonged loud reading on normal adolescent male voices. Language, Speech \& Hearing Services in Schools, 37(2), 96-103. https://doi.org/10.1044/0161-1461(2006/012)

Khoddami, S. M., Talebian, S., Izadi, F., \& Ansari, N. N. (2017). Validity and reliability of surface electromyography in the assessment of primary muscle tension dysphonia. Journal of Voice, 37(3), 386. https://doi.org/10.1016/j. jvoice.2016.09.010 
Kostyk, B. E., \& Putnam Rochet, A. (1998). Laryngeal airway resistance in teachers with vocal fatigue: A preliminary study. Journal of Voice, 12(3), 287-299. https://doi.org/10.1016/s0892-1997(98)80019-2

Laukkanen, A., Ilomäki, I., Leppänen, K., \& Vilkman, E. (2008). Acoustic measures and self- reports of vocal fatigue by female teachers. Journal of Voice, 22(3), 283-289. https://doi.org/10.1016/j.jvoice.2006.10.001

Lin, T., Chen, J., Liu, C., Lee, C., Tsou, Y., \& Chuang, C. (2017). A feasibility study on non- invasive oxidative metabolism detection and acoustic assessment of human vocal cords by using optical technique. Scientific Reports, 7(17002). https://doi.org/10.1038/s41598-017-16807-2

Lopes, L. W., da Silva, J. D., Simões, L. B., Evangelista, D. d. S., Silva, P. O. C., Almeida, A. A., \& de Lima-Silva, Maria Fabiana Bonfim. (2017). Relationship between acoustic measurements and self-evaluation in patients with voice disorders. Journal of Voice, 37(1), 119. https://doi.org/10.1016/j. jvoice.2016.02.021

Maamary, J. A., Cole, I., Darveniza, P., Pemberton, C., Brake, H. M., \& Tisch, S. (2017). Relationship between laryngeal electromyography and video laryngostroboscopy in vocal fold paralysis. Journal of Voice, 37(5), 638-642. https://doi.org/10.1016/j. jvoice.2017.02.003

Seikel, J., Drumright, D., \& Seikel, P. (2013). Essentials of anatomy \& physiology for communication disorders (2nd ed.) Delmar Cengage Learning.

Stemple, J. C., Stanley, J., \& Lee, L. (1995). Objective measures of voice production in normal subjects following prolonged voice use. Journal of Voice, 9(2), 127-133. https://doi.org/10.1016/s0892-1997(05)80245-0

Vertigan, A. E., Kapela, S. M., Franke, I., \& Gibson, P. G. (2017). The effect of a vocal loading test on cough and phonation in patients with chronic cough. Journal of Voice, 37(6), 763- 772. https://doi.org/10.1016/j.jvoice.2017.03.020

Welham, N. V., \& Maclagan, M. A. (2003). Vocal fatigue: Current knowledge and future directions. Journal of Voice, 17(1), 21-30. https://doi.org/10.1016/s08921997(O3)OOO33-x 
Whitling, S., Lyberg-Åhlander, V., \& Rydell, R. (2017a). Long-time voice accumulation during work, leisure, and a vocal loading task in groups with different levels of functional voice problems. Journal of Voice, 37(2). https:// doi.org/10.1016/j.jvoice.2016.08.008

Whitling, S., Rydell, R., \& Lyberg Åhlander, V. (2015). Design of a clinical vocal loading test with long-time measurement of voice. Journal of Voice, 29(2). https://doi.org/10.1016/j.jvoice.2014.07.012 